\title{
Increasing Stability, Efficiency, and Fundamental Understanding of Lithium-Mediated Electrochemical Nitrogen Reduction
}

\author{
Andersen, Suzanne Z.; Statt, Michael J.; Bukas, Vanessa J.; Shapel, Sarah G. ; Pedersen, Jakob B.; \\ Krempl, Kevin; Saccoccio, Mattia; Chakraborty, Debasish; Kibsgaard, Jakob; Vesborg, Peter C.K. \\ Total number of authors:
}

12

Published in:

Energy \& Environmental Science

Link to article, DOI:

10.1039/D0EE02246B

Publication date:

2020

Document Version

Peer reviewed version

Link back to DTU Orbit

Citation (APA):

Andersen, S. Z., Statt, M. J., Bukas, V. J., Shapel, S. G., Pedersen, J. B., Krempl, K., Saccoccio, M., Chakraborty, D., Kibsgaard, J., Vesborg, P. C. K., Nørskov, J., \& Chorkendorff, I. (2020). Increasing Stability, Efficiency, and Fundamental Understanding of Lithium-Mediated Electrochemical Nitrogen Reduction. Energy \& Environmental Science, 13, 4291-4300. https://doi.org/10.1039/D0EE02246B

\section{General rights}

Copyright and moral rights for the publications made accessible in the public portal are retained by the authors and/or other copyright owners and it is a condition of accessing publications that users recognise and abide by the legal requirements associated with these rights.

- Users may download and print one copy of any publication from the public portal for the purpose of private study or research.

- You may not further distribute the material or use it for any profit-making activity or commercial gain

- You may freely distribute the URL identifying the publication in the public portal 
This article can be cited before page numbers have been issued, to do this please use: S. Z. Andersen, M.

J. Statt, V. J. Bukas, S. G. Shapel, J. B. Pedersen, K. Krempl, M. Saccoccio, D. Chakraborty, J. Kibsgaard, P.

C. K. Vesborg, J. K. Norskov and I. Chorkendorff, Energy Environ. Sci., 2020, DOI: 10.1039/D0EE02246B.

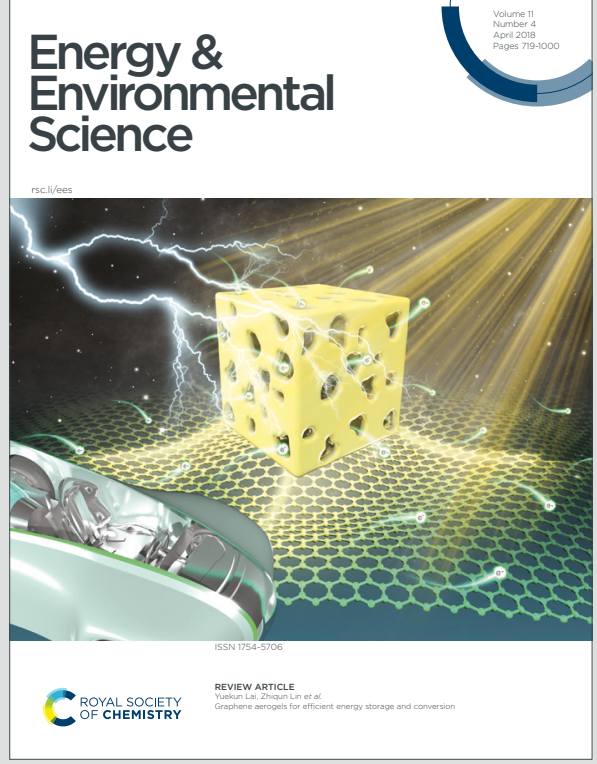

This is an Accepted Manuscript, which has been through the Royal Society of Chemistry peer review process and has been accepted for publication.

Accepted Manuscripts are published online shortly after acceptance, before technical editing, formatting and proof reading. Using this free service, authors can make their results available to the community, in citable form, before we publish the edited article. We will replace this Accepted Manuscript with the edited and formatted Advance Article as soon as it is available.

You can find more information about Accepted Manuscripts in the Information for Authors.

Please note that technical editing may introduce minor changes to the text and/or graphics, which may alter content. The journal's standard Terms \& Conditions and the Ethical guidelines still apply. In no event shall the Royal Society of Chemistry be held responsible for any errors or omissions in this Accepted Manuscript or any consequences arising from the use of any information it contains. 


\section{ARTICLE}

\section{Increasing Stability, Efficiency, and Fundamental Understanding of Lithium-Mediated Electrochemical Nitrogen Reduction}

Received 00th January 20xx, Accepted 00th January 20xx DOI: $10.1039 / \times 0 \times x 00000 x$

Suzanne Z. Andersen ${ }^{\mathrm{a}}$, Michael J. Statt ${ }^{\mathrm{b}}$, Vanessa J. Bukas $^{\mathrm{a}}$, Sarah G. Shapel ${ }^{\mathrm{a}}$, Jakob B. Pedersen $^{\mathrm{a}}$, Kevin Krempl ${ }^{a}$, Mattia Saccoccio ${ }^{a}$, Debasish Chakraborty ${ }^{a}$, Jakob Kibsgaard ${ }^{a}$, Peter C. K. Vesborga, Jens Nørskov ${ }^{a *}$, and Ib Chorkendorffa*

Lithium-mediated nitrogen reduction is a proven method to electrochemically synthesize ammonia; yet the process has so far been unstable, and the continuous deposition of lithium limits its practical applicability. One major problem of further developing this process is that very little is understood about the mechanism. We develop a model to start understanding the kinetics and we use our understanding to develop a potential cycling procedure. We show experimentally that it improves stability of the system, and leads to a significant increase in Faradaic efficiency.

\section{Introduction}

Ammonia is currently the second most produced industrial chemical, with annual production exceeding $172 \mathrm{Mt}$ [1]. It is primarily used as a fertilizer and has played a central role in sustaining the growing world population over the last century [2], [3]. Ammonia is also an essential base chemical in industry and, importantly, holds promise as a potential energy vector if production is efficiently coupled to a renewable energy source like solar or wind [4], [5]. Currently, the production of ammonia relies on the catalytic Haber-Bosch process, which thermally reduces atmospheric nitrogen with hydrogen, i.e. $\mathrm{N}_{2}$ $+3 \mathrm{H}_{2} \rightarrow 2 \mathrm{NH}_{3}$, at high temperatures $\left(350-500{ }^{\circ} \mathrm{C}\right.$ ) and pressures (150-200 bar) [6], [7]. The energy demand of this process presently amounts to $\sim 1 \%$ of the global energy consumption [8] and gives rise to significant $\mathrm{CO}_{2}$ emissions [9], [10], mainly due to the required $\mathrm{H}_{2}$ production via steam-reforming of fossil fuels [11]. Additionally, the needed high-pressure reaction conditions call for large centralized facilities with a large cost of installation and additional cost for transporting ammonia to the point of use.

The carbon footprint of hydrogen production for the Haber-Bosch process can be reduced substantially by electrifying the heating in the steam reforming process [11] or by using water electrolysis. Alternatively, a direct electrochemical synthesis of ammonia, i.e.

\footnotetext{
a. Department of Physics, Technical University of Denmark, Kongens Lyngby, Denmark.

b. SUNCAT Center for Interface Science and Catalysis, Department of Chemical Engineering, Stanford University, Stanford, CA, USA

* Corresponding author emails: jkno@dtu.dk ibchork@fysik.dtu.dk

† These authors have contributed equally

Electronic Supplementary Information (ESI) available: [details of any supplementary information available should be included here]. See DOI: 10.1039/x0xx00000x
}

$\mathrm{N}_{2}+6 \mathrm{H}^{+}+6 \mathrm{e}^{-} \rightarrow 2 \mathrm{NH}_{3}$, presents an extremely attractive substitute to the Haber-Bosch process [8], [12]. Such a synthesis route can, in principle, operate under mild conditions of $<100^{\circ} \mathrm{C}$ and near-atmospheric pressure, while simultaneously reducing our dependence on fossil-based hydrocarbons in ammonia production. This would provide a clean and sustainable $\mathrm{NH}_{3}$ supply, and enable on-site small-scale production of fertilizers which, in turn, circumvents the need for transport/storage and mitigates further environmental issues, such as runoff-water pollution through nitrate contamination [7].

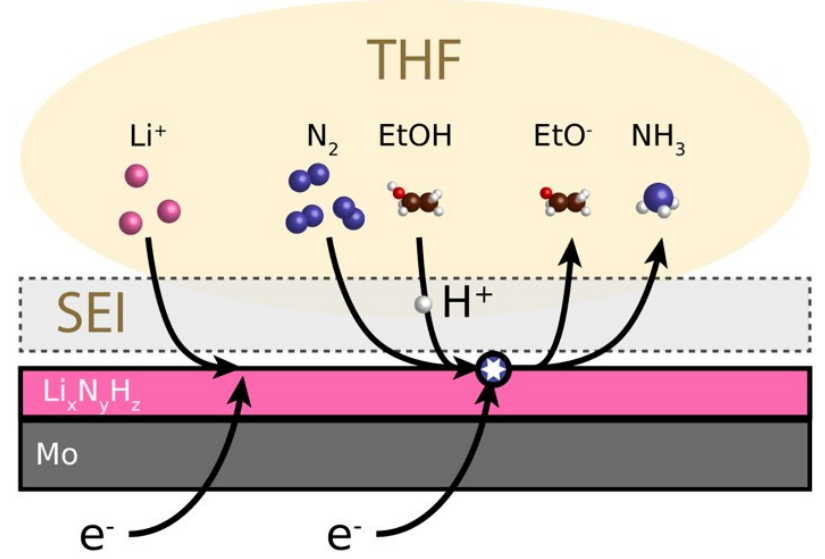

Figure 1 Schematic of the mechanism for lithium-mediated $\mathrm{N}_{2}$ electroreduction to $\mathrm{NH}_{3}$. A non-aqueous electrolyte (here, THF) contains lithium salt which is electrodeposited onto a metal electrode (here, Mo) as metallic lithium. The latter spontaneously breaks the triple $\mathrm{N}_{2}$ bond and, in the presence of an appropriate proton source (here, ethanol), this is hydrogenated to form ammonia. The exact 
composition and structure of the lithium-containing layer is not fully known.

The electrochemical $N_{2}$ reduction reaction (eNRR) faces two main challenges that currently limit its practical application. Firstly, while the ambient reaction is overall thermodynamically favorable at a potential of $0.07 \mathrm{~V}$ vs RHE, activating the inert $\mathrm{N}_{2}$ molecules makes the process extremely demanding. Experiments producing significant amounts of ammonia suffer from very large overpotentials, for an overview, please see Reference [13]. Secondly, eNRR always has to compete against the much more facile hydrogen evolution reaction (HER) [14]-[16]. It has been suggested [16] that the competition against hydrogen evolution can be suppressed through the use of a non-aqueous solvent [17], which limits the activity of protons at the cathode surface. Using a tetrahydrofuran (THF)-based solvent, a lithium-mediated strategy reports some of the highest reproducible efficiencies toward electrochemical $\mathrm{NH}_{3}$ synthesis to date. It was presented as early as 1931 by Fichtner et al. [18], then again in 1993 and 1994 by Tsuneto et al. [19], [20] and confirmed via quantitative isotope labelled studies in 2019 [13]. This method involves reducing lithium ions to lithium metal whose reactivity is unique among other alkali metals in that it can spontaneously split the $\mathrm{N}_{2}$ triple bond at ambient conditions. Subsequent reaction with a proton source can then form ammonia as schematically shown in Figure 1. The lithiummediated process works at ambient pressure with a Faradaic efficiency (FE) of up to $19 \%$ at a current density of $-8 \mathrm{~mA} / \mathrm{cm}^{2}$ [21] and, according to another study, with up to $\sim 29 \% \mathrm{FE}$ at a current density of $-2 \mathrm{~mA} / \mathrm{cm}^{2}$ and 10 bar $\mathrm{N}_{2}$ pressure [20].

While promising, the lithium-mediated strategy is still far from practical for application. The strongly reducing potentials needed to plate Li lead to electrode/electrolyte degradation and instability over time [19], while the requirement for continuously replenishing the electrolyte with $\mathrm{Li}$ salts limits the scalability of the process. Continuous Li deposition at approximately $-3.0 \mathrm{~V}$ vs SHE also greatly reduces the overall energy efficiency. The highest reported value to date is approximately $1.5 \%$ for a 40 minute experiment [21], and $2.8 \%$ for a 6 min experiment [22], far lower than what could be considered economically viable. One of the main obstacles to optimizing this process is that there is no clear understanding of the mechanism.

In their seminal studies of 1993, Tsuneto et al. proposed that deposited Li reacts with $\mathrm{N}_{2}$ to form $\mathrm{Li}_{3} \mathrm{~N}$ which is then reduced by an appropriate protic additive such as ethanol (EtOH) toward ammonia [19]. This last step was suggested to occur alongside the simultaneous release of EtOLi (i.e. as $\mathrm{Li}_{3} \mathrm{~N}$ ethanolysis) which returns at least some fraction of the deposited Li back into solution. Only very recently, Lazouski et al. made significant progress by developing a microkinetic model that introduces transport limitations into the mechanism [21]. This model, however, relied upon the same key assumption that metallic $\mathrm{Li}$ is being consumed during catalytic conversion to either $\mathrm{H}_{2}$ or $\mathrm{NH}_{3}$. While this may be the case under specific reaction conditions, such as significant build-up of a nonconductive phase on the electrode, it is not clear why Li dissolution should be a generally required step of the mechanism. The electrons

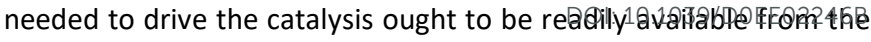
underlying metallic electrode when operating at Li-reducing potentials, therefore leaving the surface Li intact. Indeed, Schwalbe et al. most recently reported tens of nanometers of deposited $\mathrm{Li}$ coverage, while measuring similarly high values of $~ 10 \% \mathrm{FE}$ toward ammonia [23]. In absence of any electrochemical or mechanical driving force toward dissolution, the fate and mechanistic role of accumulated $\mathrm{Li}$ at the electrode remains unknown. From all the above, it is clear that a more general model is needed to understand and optimize the Li-mediated eNRR mechanism.

The purpose of the present report is two-fold. Firstly, we present a general molecular-level model to describe the mechanism of lithiummediated $\mathrm{N}_{2}$ reduction to $\mathrm{NH}_{3}$. Despite its simplicity, this model can explain a number of mechanistic features that have been observed experimentally, such as the dependence of $\mathrm{FE}$ upon $\mathrm{N}_{2}$ partial pressure and $\mathrm{EtOH}$ concentration. Based on this understanding, we develop a new strategy for increasing the FE by cycling the applied potential between a regime where Li deposition is favored and one where Li dissolution is favored. This strategy leads to a significant improvement to both the system's stability and catalytic efficiency. We show experimentally that the Li cycling process is stable for up to (at least) 125 hours of operation and yields a substantial increase in FE from $\sim 21 \%$ during constant Li deposition to $\sim 37 \%$ during potential cycling. Importantly, we additionally measure an energy efficiency of $\sim 7 \%$ that is well beyond the previously highest reported value of $2.8 \%$ in the literature for a short-term 6 minute experiment [22].

\section{Stability of the Li mediated electrochemical $\mathbf{N}_{\mathbf{2}}$ reduction process}

By way of motivating the present work, consider one of the main practical challenges with a Li-mediated eNRR during constant deposition. As illustrated in Figure 2, and also reported by others for a similar system [19], [21], the working electrode (WE) potential slowly becomes more negative over time while applying a constant current that continuously reduces lithium, while the counter electrode (CE) potential stays constant. Figure 2 specifically shows the time-evolution of three separate experiments that continuously deposit Li at constant $-2 \mathrm{~mA} / \mathrm{cm}^{2}$ current density over a roughly twohour period at 10 bar $\mathrm{N}_{2}$ in an electrochemical autoclave [24]. Data showing ammonia concentration and amount of ammonia measured for each repeated experiment are reported in Table S1. Clearly, the system is not stable. This instability is most likely related to the gradual build-up of a thick Li-containing passivation layer that is observed on the electrode at the end of these experiments (see Figures S1-S10). We therefore tentatively attribute it to one or more of the following: I) Reduction of unwanted species that originate from decomposition of the organic electrolyte over time, II) Thickening and possible break-down of the SEI layer with continued Li plating, and III) Hindered electron transport to the catalytically active surface sites due to either a growing mechanical instability or build-up of a non-conductive bulk phase on the electrode. A more 
fundamental understanding of these processes and a definitive explanation for the cathodic drift in the WE potential of these experiments is, however, subject to a more detailed future investigation.

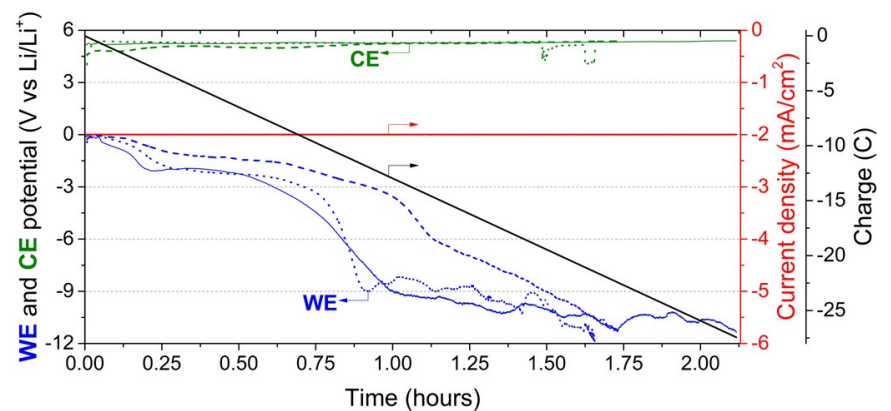

Figure 2 Constant current deposition at $-2 \mathrm{~mA} / \mathrm{cm}^{2}$ (red), for a variable total charge passed (black). The WE potentials (blue) drifts more negative over time, increasing the energy input required to sustain the desired current density. The CE potentials (green) are relatively stable. The solid, dashed, and dotted lines are three identical experiments, with similar behavior. The measurements stop when the WE potential becomes too negative, and the potentiostat overloads. Applying even higher potentials does not appear to resolve the problem [22].

These experiments yielded a mean FE of $21.2 \pm 1.6 \%$ (identical experiments repeated $\mathrm{n}=3$ times). A similar experiment from a previous report at 1 bar yielded a FE of $7.5 \pm 1.1 \%$ [13], showing a three-fold increase in Faradaic efficiency with increased $\mathrm{N}_{2}$ pressure. A similar effect has been observed by Tsuneto et al. [20] for increased pressure up to 50 bar, and Lazouski et al. who reported a linear increase in $\mathrm{FE}$ for $\mathrm{N}_{2}$ partial pressures between 0 and 1 bar [21].

The energy efficiency of the deposition experiments at 10 bar is calculated to be $2.3 \pm 0.4 \%(n=3)$. The energy efficiency is determined based on the report by Lazouski et al. [21] (calculation stated in the $\mathrm{SI}$ ), as only they report an energy efficiency in this field. Our experiments are however carried out at 10 bar $\mathrm{N}_{2}$ in a single compartment cell. The report by Lazouski et al. uses EtOH as a sacrificial proton source, but does not account for this loss in their energy efficiency. Since we want as direct a comparison as possible, we also do not account for the use of sacrificial EtOH in our energy efficiency calculations. Additionally, we do not include the energy needed to pressurize the cell.

It should be mentioned that there are substantial energy losses connected to the anode in the single compartment cell. Ideally, a two-compartment setup with a membrane should be used for better control of the anode reaction [21], which would then allow for the use of $\mathrm{H}_{2}$ and the hydrogen oxidation reaction [22], rather than sacrificial EtOH as a proton source. Obviously, the non-stable situation could result in the low Faradaic efficiency, and therefore a model providing deeper insight could be very helpful.

\section{An atomistic kinetic model of eNRR View Article Online DOI: 10.1039/DOEE02246B}

We first develop a simple kinetic model to describe the mechanism of Li-mediated $\mathrm{N}_{2}$ reduction and the resulting FE toward $\mathrm{NH}_{3}$. The goal is to make the model as simple as possible, yet complex enough to begin understanding the observed variations in rate and FE with external conditions, and to use it to begin optimizing the process. We consider the following elementary steps occurring during Li deposition:

$$
\begin{aligned}
& L i^{+} \underset{\text { slow }}{\stackrel{r_{L i}}{\longrightarrow}} L i_{(s)}^{+} \\
& H^{+} \underset{\text { slow }}{\stackrel{r_{H}}{\rightarrow}} H_{(s)}^{+} \\
& N_{2} \underset{\text { slow }}{\stackrel{r_{N_{2}}}{\rightleftarrows}} N_{2}(s) \\
& L i_{(s)}^{+}+e^{-} \underset{\text { fast }}{\stackrel{k_{L i}}{\rightleftarrows}} L i \cdot \\
& L i \cdot+\left(H_{(s)}^{+}+e^{-}\right) \underbrace{\stackrel{k_{H-1}}{\longrightarrow}}_{\text {fast }} L i \cdot H^{*} \\
& L i \cdot H^{*}+\left(H_{(s)}^{+}+e^{-}\right) \underbrace{\stackrel{k_{H-2}}{\longrightarrow}}_{\text {fast }} L i \cdot+H_{2} \\
& L i \cdot+1 / 2 N_{2}(s) \underset{\text { fast }}{\stackrel{k_{N-1}}{\longrightarrow}} L i \cdot N^{*}
\end{aligned}
$$

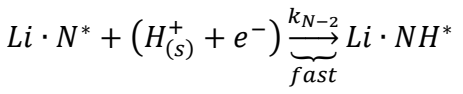

$$
\begin{aligned}
& L i \cdot N H^{*}+\left(H_{(s)}^{+}+e^{-}\right) \underset{\text { fast }}{\stackrel{k_{N-3}}{\longrightarrow}} L i \cdot N H_{2}^{*} \\
& L i \cdot N H_{2}^{*}+\left(H_{(s)}^{+}+e^{-}\right) \underset{\text { fast }}{\stackrel{k_{N-4}}{\longrightarrow}} L i \cdot+N H_{3}
\end{aligned}
$$

In the above reactions, the subscript (s) is used to note close-tosurface species, $\mathrm{Li} \cdot$ denotes an active surface site of $\mathrm{Li}$, and $\mathrm{Li} \cdot \mathrm{X}^{*}$ is a surface-bound $X$ species. All rate constants are given by $k_{x}$. Steps (1a)-(1b) overall describe Li deposition, while steps (2a)-(2c) and (3a)-(3e) describe the catalytic formation of $\mathrm{H}_{2}$ and $\mathrm{NH}_{3}$, respectively. Note that $\mathrm{Li} \cdot$ is not consumed in any step, which implies that lithium should continuously plate and build up on the electrode over the course of the deposition period.

We explicitly consider the diffusion of $\mathrm{Li}^{+}, \mathrm{H}^{+}$and $\mathrm{N}_{2}$ species from bulk solution and toward the surface. These diffusion steps are most likely significantly slower under these experimental conditions relative to aqueous conditions due to the presence of the 'protective' passivation layer that has been shown to form at the solid electrolyte interphase (SEI) of Li ion batteries during charging (i.e. Li deposition) [25]. We will assume in the following that reactions (1a), (2a), and (3a), with rates $r_{L i}, r_{H}$, and $r_{N_{2}}$ respectively, 
are overall rate-limiting. We will further assume that the initial deposition of $\mathrm{Li}$ is fast enough so that steady-state conditions are quickly established. This assumption is in accordance with the experimental data shown later in Figure 5.

Relative to the slow diffusion steps, the subsequent electrochemical steps are expected to be very fast due to the extreme reducing potentials required to plate Li. Following the arguments of Singh et al. [26], we assume that such electrochemical steps, (1b), (2b)-(2c), and (3c)-(3e) above, have negligible activation energies and are driven irreversibly forward. We make the same assumption for the dissociative $\mathrm{N}_{2}$ chemisorption on metallic Li (step 3b), which is not an electrochemical step, but has been calculated to have a very low kinetic barrier [27].

When diffusion to the surface is rate-limiting, there will be limited availability of $N_{2(s)}$ and $H_{(s)}^{+}$species, and that establishes the following species balances for hydrogen and nitrogen under steady state conditions:

$$
\begin{gathered}
r_{H}=2 r_{\mathrm{H}_{2}}+3 r_{\mathrm{NH}_{3}} \\
r_{\mathrm{NH}_{3}}= \begin{cases}2 r_{N_{2}} & r_{N_{2}} \leq \frac{1}{6} r_{H} \\
\frac{1}{3} r_{H} & r_{N_{2}} \geq \frac{1}{6} r_{H}\end{cases}
\end{gathered}
$$

Equation (4) simply balances the incoming protons with outgoing hydrogen and ammonia, i.e. the assumption being that all protons are consumed to form either $\mathrm{H}_{2}$ or $\mathrm{NH}_{3}$ at steady state. Equation (5) then expresses the rate of $\mathrm{NH}_{3}$ production as a piecewise function that results from the two possible mass-transfer limitations: either a limitation in nitrogen, or a limitation in protons. When the rate of proton arrival is much greater (more than six times) than the rate of nitrogen arrival, all incoming nitrogen is assumed to balance the outgoing ammonia. On the other hand, when proton transfer is much slower (less than six times) than nitrogen transfer, the rate of $\mathrm{NH}_{3}$ production is tied to the rate of incoming protons. In this regime, excess nitrogen accumulating on the surface may lead to build-up of a bulk lithium nitride phase.

Using these simple transport-limited reactions, we can formulate the FE during Li deposition as a function of the three $\left(r_{L i}, r_{H}, r_{N_{2}}\right)$ diffusion rates:

$$
\begin{aligned}
F E_{\text {deposition }}= & \frac{3 r_{N H_{3}}}{3 r_{N_{3}}+2 r_{H_{2}}+r_{L i}} \\
& = \begin{cases}\frac{6 r_{N_{2}}}{r_{H}+r_{L i}} & r_{N_{2}} \leq \frac{1}{6} r_{H} \\
\frac{r_{H}}{r_{H}+r_{L i}} & r_{N_{2}} \geq \frac{1}{6} r_{H}\end{cases}
\end{aligned}
$$

where $3 r_{\mathrm{NH}_{3}}, 2 r_{\mathrm{H}_{2}}$, and $r_{\mathrm{Li}}$ represent electron consumption toward eNRR, HER, and Li deposition respectively. The resulting denominator $\left(r_{H}+r_{L i}\right)$ is the total current which can only be passed through protons or lithium. The relationship between

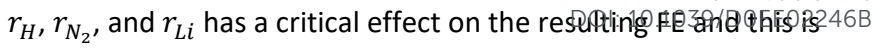
graphically represented (via Eq. 6) in Figure 3(a).
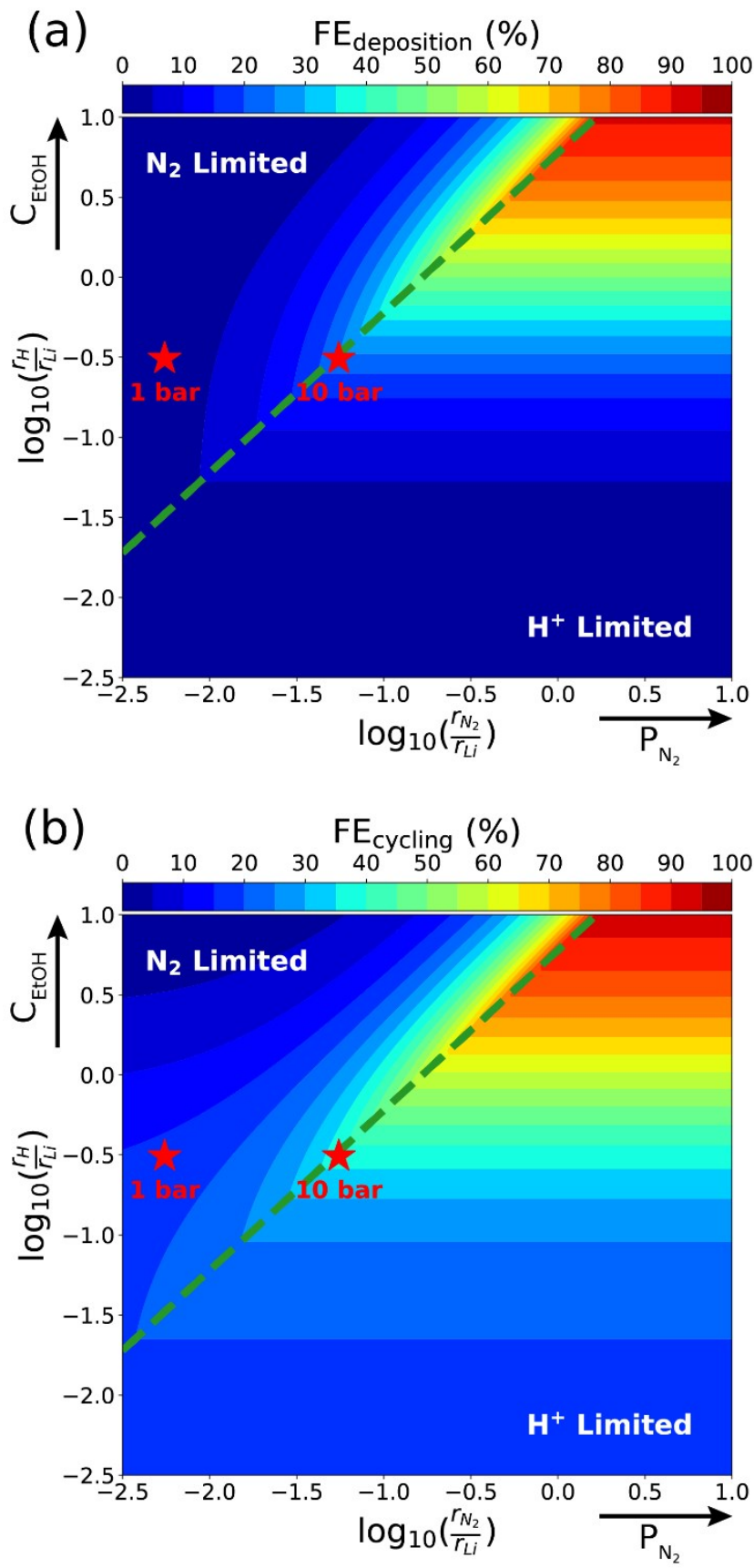

Figure 3: Heatmap of the predicted FE as a function of the ratio of nitrogen to lithium (x-axis) and proton to lithium (y-axis) diffusion rates. (a) shows the FE during constant Li deposition (using Eq. (6)), while (b) shows the FE during potential cycling (using Eq. (8)). In both cases, the green line indicates the boundary between regimes of $\mathrm{N}_{2}$ transport limitations (upper left) and $\mathrm{H}^{+}$transport limitations (lower right). The stars have been specifically placed to show where our experimentally measured FE values at 1 and 10 bar would fall on this plot (see SI for details). The arrows beside the vertical and horizontal axes indicate how increases in ethanol concentration $\left(\mathrm{C}_{\mathrm{EtOH}}\right)$ and 
nitrogen pressure $\left(P_{N_{2}}\right)$ will correspond to vertical and horizontal movements, respectively.

Here, the boundary between the nitrogen- and proton-limited regimes $r_{N_{2}} / r_{H}=1 / 6$ is outlined by the dashed green line and shown to correspond to the ideal ratio of $\mathrm{N}_{2}$ vs. $\mathrm{H}^{+}$impingement. Below this line eNRR is limited by proton diffusion, while the region above the line is limited by nitrogen diffusion. It is clear that when the rate of proton arrival is strongly mismatched to that of nitrogen arrival or either rate is significantly lower than the Li diffusion rate, the $\mathrm{FE}$ is near $0 \%$. The strong dependence on transport of these different species through the electrolyte and SEI suggests that experimental conditions will strongly determine the overall rate and FE toward forming ammonia.

Within the framework of the model, one can deduce key insights into the effect of experimental conditions on FE. However, to do so we must first understand the regime in which a set of experiments operates. To this end, we fit experimental values of FE at given nitrogen pressures $\left(\mathbf{P}_{\mathbf{N}_{2}}\right)$ and ethanol concentrations $\left(\mathrm{C}_{\mathrm{EtOH}}\right)$. During fitting, $\mathbf{P}_{\mathbf{N}_{2}}$ and $\mathrm{C}_{\mathrm{EtOH}}$ are assumed to be directly proportional to $r_{N_{2}} / r_{L i}$ and $r_{H} / r_{L i}$, respectively. As a result, we utilize two fitted conversion factors to relate $P_{N_{2}}$ to $r_{N_{2}} / r_{L i}$ and $C_{\text {EtoH }}$ to $r_{H} / r_{L i}$ (see $\mathrm{SI}$ for fitting details). The comparison of the resulting model to experimental data is shown in Figures 3(a) and 4.

From these results, we observe that the 1 bar experiments operate in the nitrogen-limited regime, while the 10 and 20 bar experiments operate near or in the proton-limited regime. This explains why the large increase in FE seen from 1 to 10 bar is not seen when pressure is increased to 20 bar. This effect of increasing $\mathrm{P}_{\mathrm{N} 2}$ is visualized in Figure 3(a) as a horizontal movement to the right, as this increases $r_{N_{2}}$ independently of $r_{H}$ and $r_{L i}$. According to our model, such an increase in $r_{N_{2}}$ will only improve the overall FE in the regime where $\mathrm{N}_{2}$ is the limiting reagent. As Lazouski et al. reported a linear increase in FE for $\mathrm{N}_{2}$ partial pressures between 0 and 1 bar [21], it is likely that their system still operated entirely within the $\mathrm{N}_{2}$ limited regime. Our model reproduces this linear dependence within the nitrogenlimited regime as clearly shown by Figure 4(a) which plots $\mathrm{FE}_{\text {deposition }}$ as a function of $r_{N_{2}} / r_{L i}$ at a fixed $r_{H} / r_{L i}=0.31$ ratio that is matched to our experimentally measured FEs (cf. below). Once eNRR transitions over to the proton-limited regime, however, the dependence of $\mathrm{FE}$ on $\mathrm{N}_{2}$ pressure should be lost. This is reflected here by the $\mathrm{FE}_{\text {deposition }}$ plateau at $r_{N_{2}} / r_{H}>1 / 6$ in Figure 4 (a) (or, equivalently, through the horizontal contours that dominate the right-hand part of Figure 3(a)).

Overlaying our measured data points for FE onto Figure 4(a) shows a smoother transition between the two transport-limited regimes, which is likely due to experimental inhomogeneities across the surface not captured by our simple model. $A \pm 50 \%$ uncertainty in the nitrogen and proton diffusion ratios is included to emulate this inhomogeneity. The resulting median FE and standard error when this uncertainty is included are shown by the dashed lines and shaded regions in Figure 4, respectively. The median FE curve exhibits the smoother transition between the two limiting regimes. Additionally, the median FE curve is consistentpy1 Rowerothan2the analytical FE curve near the peak FE, as the median FE curve samples the regions of lower FE above and below the peak. We chose to fit the experimental data to the analytical FE, rather than the median $\mathrm{FE}$, as the heterogeneity of the surface has yet to be fully investigated. It should be noted that a similar saturating behavior of FE with $\mathrm{N}_{2}$ pressure was also observed in the experiments of Tsuneto et al. for higher partial pressures of $>10$ bar [20].

(a)

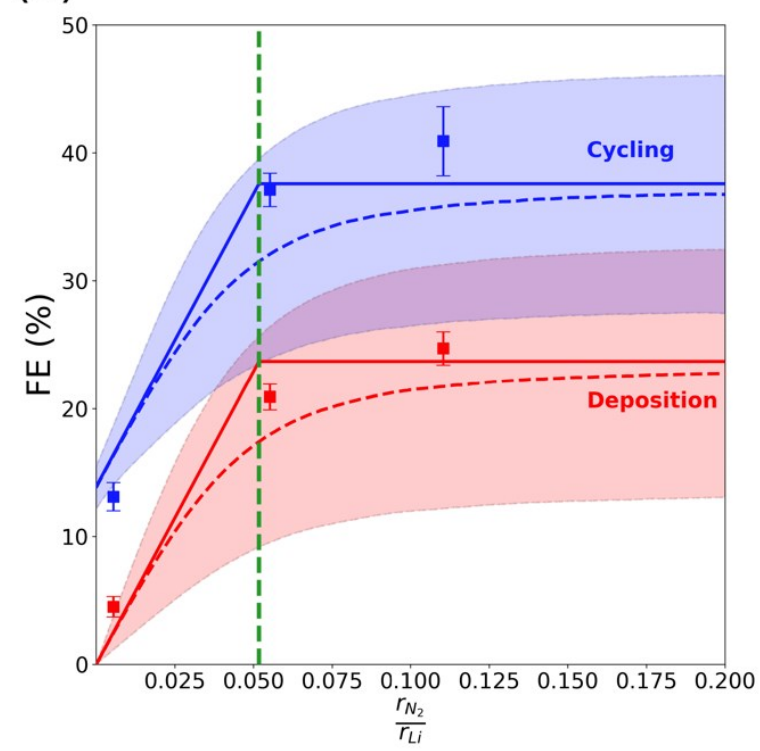

(b)

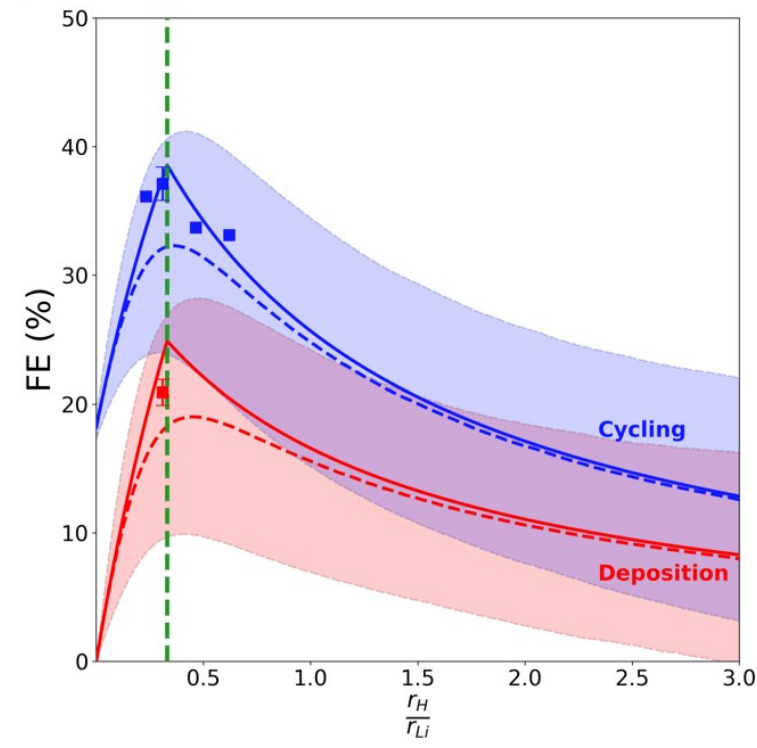

Figure 4: Predicted FE as a function of the ratio of (a) nitrogen to lithium and (b) proton to lithium diffusion rates. Red and blue lines show the FE during constant Li deposition and potential cycling respectively, while the dashed green line indicates the boundary between regimes of $\mathrm{N}_{2}$ and $\mathrm{H}^{+}$transport limitations. The shaded region surrounding each curve illustrates the spread of FE as a result of $\pm 50 \%$ uncertainty in the ratio of diffusion rates for nitrogen and 
hydrogen. Panels (a) and (b) consider fixed ratios of $r_{H} / r_{L i}=0.31$ and $r_{N_{2}} / r_{L i}=0.055$ respectively, as derived from experimentally fit conversion factors (see SI for details). The square markers in panel (a) show experimental FE measurements for estimated values of $r_{N_{2}} / r_{L i}$ at 1, 10, and 20 bar, with all data included in Tables S1-S3. The square markers in panel (b) show experimental FE measurements for estimated values of $r_{H} / r_{L i}$ at $0.75,1,1.5$, and 2 vol\% EtOH, with data reported in Tables S1 and S4. Error bars are calculated from 3 identical experiments $(n=3)$.

The effect of varying the concentration of $\mathrm{EtOH}$, and hence the availability of protons, would result in a vertical movement in Figure 3(a) where now $r_{H}$ is controlled independently of $r_{N_{2}}$ and $r_{L i}$. This clearly results in an optimum value for $\mathrm{EtOH}$ concentration at $r_{N_{2}} / r_{H}=1 / 6$. Increasing proton activity past this optimum pushes eNRR into the $\mathrm{N}_{2}$ limited regime and increases the rate of $\mathrm{H}_{2}$ production without any increase in the rate of $\mathrm{NH}_{3}$ production. This peak behavior in $\mathrm{EtOH}$ is clearly shown in the deposition curve of Figure $4(\mathrm{~b})$ which plots $F E$ as a function of $r_{H} / r_{L i}$. Similar to Figure 4(a), Figure 4(b) uses a fixed ratio for $r_{\mathrm{N}_{2}} / r_{L i}$ of 0.055 , which corresponds to 10 bar of nitrogen pressure prior to conversion through experimentally fitted parameters (see SI for details). Such a dependence of the FE with ethanol concentration has been consistently reported in multiple experimental studies [20], [21], [23].

\section{An electrochemical potential-cycling strategy}

We now turn our efforts toward improving the system's performance. Once the catalyst surface is fully covered by reduced $\mathrm{Li}$ species, any additional Li that is reduced consumes an electron and thus contributes to lowering the FE. This explains why large FEs appear only at relatively high ratios $r_{N_{2}} / r_{L i}$ and $r_{H} / r_{L i}$ in Figure 3(a), as lithium is being deposited at a lower rate relative to nitrogen and hydrogen consumption in this region. While these non-catalytic electrons are stored in the excess $\mathrm{Li}$ at the surface, they are not necessarily irrecoverable. Ideally, all high-energy electrons could be recovered by restoring the Li salt concentrations from the surface to the electrolyte. This should additionally serve the important purpose of reducing the overall resistance of the cell and avoid the need of continuously adding Li salts during operation. Finally, Li dissolution should prevent continued side reactions with the organic solvent which are known to not only lower lithium's cycling efficiency, but also consume both Li metal and the electrolyte [28].

If the extreme reducing potential is removed after a period of $\mathrm{Li}$ deposition to allow for the cathode to "rest", high energy electrons are no longer readily available to reduce incoming nitrogen or protons. However, high energy electrons stored in excess lithium on the surface may be donated to run either reaction. This couples $\mathrm{Li}$ dissolution to the two reduction reactions, replacing $\left(H_{(s)}^{+}+e^{-}\right)$ with $\left(H_{(s)}^{+}+L i \cdot\right)$ and adding a $\mathrm{Li}^{+}$to the products in steps $(2 \mathrm{~b})-$ (3e) above. This essentially uses the excess $L i$ as a pseudo-battery to run the reduction reactions (see SI for detailed mechanism). Since these electrons are effectively stored at the reduction potential of

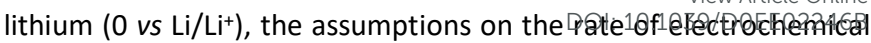
reactions being much faster than diffusion of nitrogen and protons should still hold. Thus, some fraction of the electrons used to plate Li during deposition can be recovered to selectively produce ammonia. This motivates repeated deposition-resting cycles to improve the efficiency of the Li-mediated process. Such a potential cycling approach has been used in the past to maintain stability of electrochemical systems [29]-[31], prevent electrocatalyst poisoning [32], or selectively promote specific reaction mechanisms [33]. Here, this concept is used to store and subsequently release high-energy electrons in order to continuously produce ammonia throughout the cyclic process.

We can include the effect of this potential cycling on the overall FE. For this purpose, we use $S_{\mathrm{Li} \rightarrow \mathrm{NH}_{3}}$ to denote the fraction of $\mathrm{Li}$ that is ultimately recovered toward forming ammonia. If the rate of $\mathrm{Li}$ deposition is assumed to be constant over the course of the deposition period, $t_{d}$, and the resting period is long enough for all $\mathrm{Li}$ to dissolve, the amount of ammonia produced during the resting period is simply:

$$
N_{N H_{3}}=\frac{1}{3} t_{d} r_{L i} S_{L i \rightarrow N H_{3}}
$$

Since electrons are only transferred across the electrode during deposition, the effect of this increased ammonia production on the $\mathrm{FE}$ is to simply add a constant to the numerator of equation (6):

$$
F E_{\text {cycling }}= \begin{cases}\frac{6 r_{N_{2}}+r_{L i} s_{L i \rightarrow N H_{3}}}{r_{H}+r_{L i}} & r_{N_{2}} \leq \frac{1}{6} r_{H} \\ \frac{r_{H}+r_{L i} s_{L i \rightarrow N H_{3}}}{r_{H}+r_{L i}} & r_{N_{2}} \geq \frac{1}{6} r_{H}\end{cases}
$$

where the deposition time $t_{d}$ drops out of the equation since all terms are proportional to it and the factor of $1 / 3$ drops out due to three electrons being transferred per ammonia molecule. Equation (8) reflects an obvious improvement in $F E_{\text {cycling }}$ as compared to $F E_{\text {deposition }}$ (Eq. 6) that is easily visualized in Figures 3 and 4 . These show the improvement from cycling as a constant, overall increase in FE (regardless of the relative $r_{H} / r_{L i}, r_{N_{2}} / r_{L i}$ rates) which results from the increased fraction of catalytic electrons that are being made available through the excess $\mathrm{Li}$ at the surface.

The quantitative improvement in FE due to cycling is directly related to $S_{\mathrm{Li} \rightarrow \mathrm{NH}_{3}}$. This value is expected to depend strongly on various experimental parameters that define the specific reaction conditions under investigation. By fitting equation (8) to the experimental data shown in Figure 4, we estimate $S_{\mathrm{Li} \rightarrow \mathrm{NH}_{3}}=0.18$ (see SI for details). This estimate assumes that the relative $r_{H} / r_{L i}, r_{N_{2}} / r_{L i}$ rates do not change significantly upon cycling, leading to a constant $S_{\mathrm{Li} \rightarrow N \mathrm{H}_{3}}$ to represent the fraction of deposited $\mathrm{Li}$ that is used during resting to 
produce ammonia. This value for $S_{\mathrm{Li} \rightarrow \mathrm{NH}_{3}}$ was used in Figures $3(\mathrm{~b})$ and 3.

\section{Cycling experiments}

We next examine experimentally the effect of cycling the applied potential. For this purpose, we developed a protocol that consists of short deposition pulses of $1 \mathrm{~min}$ at $-2 \mathrm{~mA} / \mathrm{cm}^{2}$ followed by $3-8 \mathrm{~min}$ at $0 \mathrm{~mA} / \mathrm{cm}^{2}$. Applying short current pulses for Li deposition, with a specific resting time between each pulse, significantly prevents the WE potential from drifting more negative over time. By increasing/decreasing the resting time, we found that we could accurately decrease/increase the WE potential, and keep it stable around $-1 \mathrm{~V}$ vs. $\mathrm{Li} / \mathrm{Li}^{+}$during deposition, as seen in Figure 5(a). This is seen as a change in the slope of the charge that is being passed, which correlates with a change in the resting time, and thereby the time-average current density.
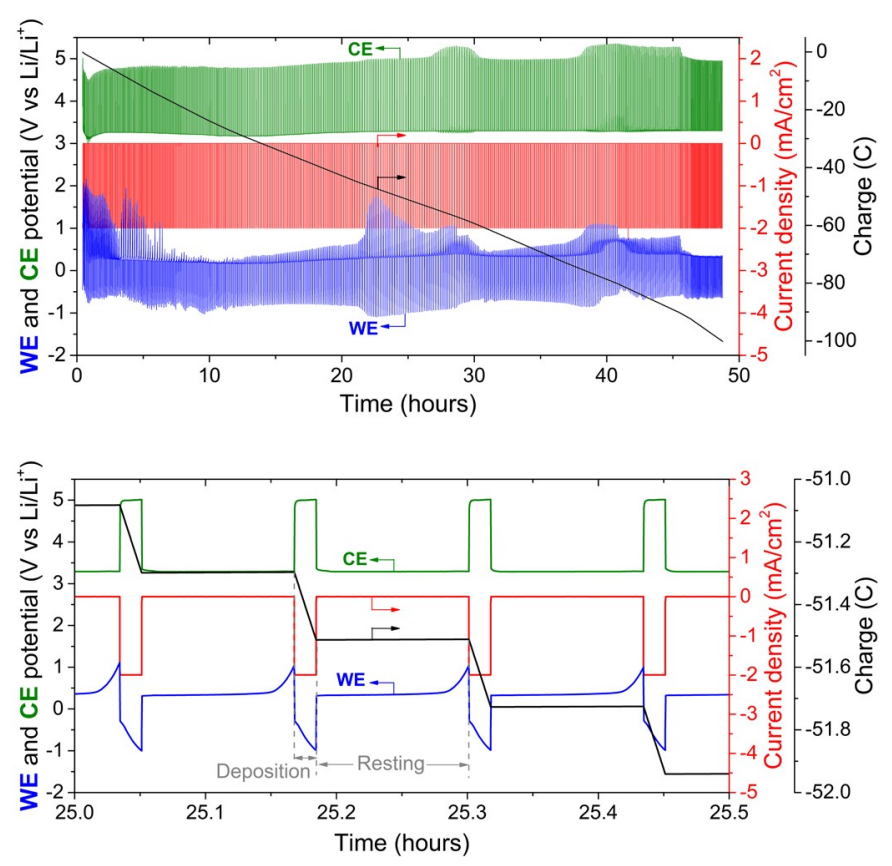

Figure 5 a) Cycling method between -2.0 and $0.0 \mathrm{~mA} / \mathrm{cm}^{2}$ (red), for a total of $100 \mathrm{C}$ of charge passed (black). The WE potential (blue) and CE potential (green) is stable across the entire experiment by varying the resting time. b) A close-up of the cycling. Immediately after switching to a deposition current, the absolute value of the WE potential increases for the entire 1 min duration. When switching back to resting, the WE potential is initially stable just above $0 \mathrm{~V} v \mathrm{vs}$. $\mathrm{Li}^{+} / \mathrm{Li}$, until it eventually starts becoming anodic after some minutes, suggesting dissolution of all surface Li species that were deposited in the preceding deposition pulse. At this point, another Li depositing pulse is applied. As the experiment did not have constant supervision, there are occasional point where the resting WE potential becomes very positive (e.g. around 22 and 40 hours) before the deposition pulse is applied. This is simply changed by shortening the resting time.

The ideal resting time was determined as the point at which the WE potential starts increasing. This is expected to occur when all previously deposited Li has been removed from the surface and was therefore followed by applying a new Li deposition pulse, as seen in Figure 5(b). This procedure corresponds well tothel hodéPab6ve? Wve checked the reproducibility of this experiment by repeating it and generating a similar CP profile, and repeating again with isotope labeled gas (discussed in the section "control experiments") i.e. three repeated measurements of the Faradaic efficiency. The mean and standard deviation of the measurements had a FE of $37.1 \pm 1.3 \%$ (n $=3$ ), an energy efficiency of $6.9 \pm 0.5 \%$, and kept a stable working electrode potential even over days.

To show the effectiveness of stabilizing the WE via potential cycling, a long-term experiment spanning 125 hours and passing $180 \mathrm{C}$ was carried out. By varying the resting time, the WE potential was controlled to be in the desired low potential region across the entire experiment. This experiment used 2 vol.\% EtOH instead of 1 vol.\%, as passing such large amounts of charge would significantly impact the $\mathrm{EtOH}$ concentration throughout the experiment. This experiment had a FE of $33.1 \pm 1.0 \%$, and an energy efficiency of $5.3 \pm 0.2 \%$ ( $n=$ 1). We speculate the slightly lower yield is due to the increase in EtOH concentration which, according to Figures $3(\mathrm{~b})$ and $4(\mathrm{~b})$, suggests that the ideal rate of proton transfer was somewhat overshot (i.e. corresponding to a too-large vertical upward movement in Figure 3(b)). Despite this, the experiment still had a much higher FE and energy efficiency compared to the constant deposition case.

\section{Control experiments}

Although the lithium-mediated method has already previously been proven to synthesize ammonia, we followed the rigorous isotope labelled electrochemical nitrogen reduction protocol previously published [13] to further validate our results: we performed first $\mathrm{Ar}$ blank experiments, next $\mathrm{N}_{2}$ experiments at OCP, and finally an isotope labelled experiment using ${ }^{15} \mathrm{~N}_{2}$. Using the protocol mentioned in reference [13], we have tested for hydrazine as a possible side product, but do not measure any in this system.

Ar blank and $\mathbf{N}_{\mathbf{2}}$ at OCP For Ar blank experiments, with $100.7 \mathrm{C}$ passed, a background of $0.5 \pm 0.1$ p.p.m. of ammonia was measured using indophenol. NMR on a single sample gave a concentration of 0.4 p.p.m. of ${ }^{14} \mathrm{NH}_{3}$ for comparison, seen in Figure 6. For the 24 -hour $\mathrm{N}_{2}$ experiments at OCP, with pre-purging of the electrolyte with cleaned $\mathrm{N}_{2}$ gas, a concentration of $0.4 \pm 0.1$ p.p.m of contamination was measured. We inherently have a high level of contamination in our system due to the amounts of ammonia produced in regular experiments (sometimes above 100 p.p.m. in $30 \mathrm{~mL}$ electrolyte), which will stick to the autoclave walls and pipes, and is unfortunately hard to get rid of. However, as we are producing orders of magnitude more ammonia during electrochemistry compared to our background, the sub-p.p.m. background contamination is insignificant in comparison, and is simply subtracted from the total.

Quantitative isotope experiments As the lithium-mediated process has already been proven to synthesize ammonia [13], only a single isotope labeled experiment was done to verify the increase in FE for the newly developed cycling method. The autoclave volume is 
$380 \mathrm{~cm}^{3}$, and experiments are all at 10 bar $\mathrm{N}_{2}$, meaning we could not fill up the entire autoclave with ${ }^{15} \mathrm{~N}_{2}$, as the bottles are $416 \mathrm{~mL}$, and contain a total of $5 \mathrm{~L}$ of gas. For this reason, we aimed at utilizing a mixed composition gas of ${ }^{14} \mathrm{~N}_{2}$ and ${ }^{15} \mathrm{~N}_{2}$, and confirmed via massspectroscopy that $78 \pm 2$ vol.\% ${ }^{14} \mathrm{~N}_{2}$ and $22 \pm 2$ vol.\% ${ }^{15} \mathrm{~N}_{2}$ was achieved.

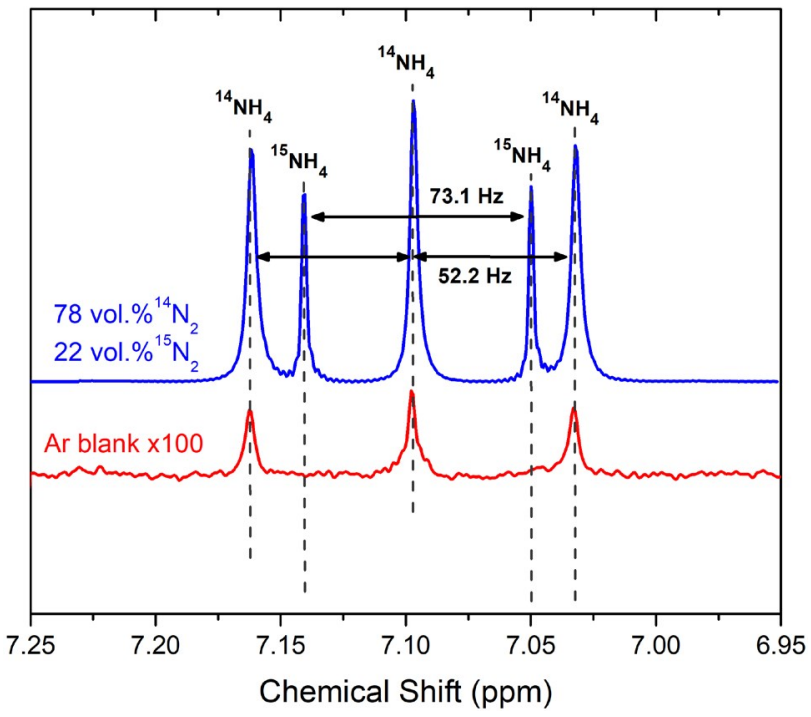

Figure 6 NMR data from using a previously developed THF suppression method [13], [34]. The red curve is an Ar blank sample, magnified by 100 to show the spectra in comparison to the blue curve, which is the isotope labelled experiment at 10 bar with potential cycling with a combination of ${ }^{15} \mathrm{~N}_{2}$ and ${ }^{14} \mathrm{~N}_{2}$ gas.

From the NMR sample seen in Figure 6, we measured an ${ }^{15} \mathrm{NH}_{3}$ concentration of 15.6 p.p.m. and an ${ }^{14} \mathrm{NH}_{3}$ concentration of 67.1 p.p.m, totaling 82.6 p.p.m., with 82 rel. ${ }^{14} \mathrm{NH}_{3}$ to 18 rel.\% ${ }^{15} \mathrm{NH}_{3}$. The non-isotope-sensitive indophenol measurement gave $81.3 \pm$ 4.2 p.p.m., in perfect accordance with the NMR. The FE of this measurement was $37.6 \pm 2.0 \%$ with an energy efficiency of $6.5 \pm$ $0.3 \%$ for $100 \mathrm{C}$ charged passed. This compares well with the nonisotope sensitive experiments carried out in Figure 5, which has now been repeated a total of 3 times with the cycling method (twice with ${ }^{14} \mathrm{~N}_{2}$ and once with a mixture of ${ }^{14} \mathrm{~N}_{2}$ and ${ }^{15} \mathrm{~N}_{2}$ ) passing $100 \mathrm{C}$ of charge each. All experimental data are reported in Table S1 and S4.

\section{The usefulness of the model and outlook}

While lithium-mediated ammonia synthesis still has significant obstacles to practical viability, such as a replacement for the sacrificial proton source, our model can be used to further improve the process. The use of gas diffusion electrodes may provide a very promising alternative to $\mathrm{N}_{2}$ pressure for independently increasing the mass transport of nitrogen relative to that of protons. The model suggests that a much higher rate at a similar FE could be obtained, but only if the nitrogen transfer rate to the surface is increased proportionally.
Our model also sheds some light on what is so special about the Li-

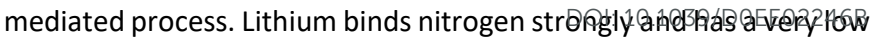
barrier toward $\mathrm{N}_{2}$ dissociation [27], but early transition metals (and nitrides) have similar behavior [14], [35]. Li is, however, the only electrode material known (so far, at least) to develop a protective SEI layer. Similar to the situation in Li ion batteries, this passivating SEI layer likely plays a vital role in determining the system's function and catalytic performance. Firstly, it may help improve the system's stability by preventing excess electrolyte degradation with organic decomposition products accumulating on the working electrode. This should not only protect the catalyst from poisoning, but also help maintain the demonstrated long-term Li cycling ability. Secondly, as already indicated above, the SEI layer is most likely a crucial factor in controlling the relative $\left(r_{L i}, r_{H}, r_{N_{2}}\right)$ diffusion rates which emerge from our model as a critical variable toward achieving a high eNRR FE. Relatively slow proton transport in particular is reminiscent of the ambient $\mathrm{N}_{2}$ electroreduction by the enzyme nitrogenase, where anaerobic conditions lead to a well-controlled proton transfer pathway through the protein and a resulting $~ 75 \% \mathrm{FE}$ toward $\mathrm{NH}_{3}[36]$.

An important step forward will be to better understand the composition of the SEI layer, as well as how each of the three diffusing species interact with it, in order to be able to tune the individual diffusion rates toward optimal conditions. Much work has been done in the battery community to develop artificial SEl's to stabilize lithium metal batteries [37]. Using solvent additives to develop an artificial SEI may allow for diffusion rates to be tuned as well as alternatives to lithium metal to be used. One could also imagine building an SEl layer on other electrode materials using that to control proton transport, thus avoiding Li deposition altogether.

\section{Conflicts of interest}

A patent application titled "Cyclic stabilization of lithium mediated electrochemical nitrogen reduction" was submitted on 05-03-2020 regarding the cyclic stabilization method mentioned in the paper. The authors declare no financial conflicts.

\section{Acknowledgements}

We gratefully acknowledge the funding by Villum Fonden, part of the Villum Center for the Science of Sustainable Fuels and Chemicals (V-SUSTAIN grant 9455). The $800 \mathrm{MHz}$ NMR spectra were recorded at the NMR center at DTU, supported by the Villum Foundation. We thank Celia Cailloux for helping with XRD, Kasper Enemark-Rasmussen for acquiring NMR spectra, and the SurfCat floor managers Brian P. Knudsen and Patrick Strøm-Hansen for setting up the mass spectrometer.

\section{References}

[1] L. E. Apodaca, "Nitrogen (fixed) - Ammonia." Mineral Commodity Summaries - U.S. Geological Survey, pp. 116- 
117, 2019.

[2] G. Ertl, "Reactions at Surfaces: From Atoms to Complexity (Nobel Lecture)," Angew. Chemie Int. Ed., vol. 47, no. 19, pp. 3524-3535, Apr. 2008.

[3] “Ammonia From Ullmann's Encyclopedia of Industrial Chemistry,7th Ed."

[4] R. F. Service, "Liquid Sunshine," Science (80-. )., vol. 361, no. 6398, pp. 120-123, 2018.

[5] C. H. Christensen, T. Johannessen, R. Z. Sørensen, and J. K. Nørskov, "Towards an ammonia-mediated hydrogen economy?," Catal. Today, vol. 111, no. 1-2, pp. 140-144, Jan. 2006.

[6] F. Haber and R. Le Rossignol, "Über die technische Darstellung von Ammoniak aus den Elementen," $Z$. Elektrochem. Angew. Phys. Chem., vol. 19, no. 2, pp. 5372, 1913.

[7] J. W. Erisman, M. a. Sutton, J. Galloway, Z. Klimont, and W. Winiwarter, "How a century of ammonia synthesis changed the world," Nat. Geosci., vol. 1, no. 10, pp. 636-639, Oct. 2008.

[8] C. J. M. van der Ham, M. T. M. Koper, and D. G. H. Hetterscheid, "Challenges in reduction of dinitrogen by proton and electron transfer," Chem. Soc. Rev., vol. 43, no. 15, pp. 5183-5191, 2014.

[9] I. Rafiqul, C. Weber, B. Lehmann, and A. Voss, “Energy efficiency improvements in ammonia productionperspectives and uncertainties," Energy, vol. 30, no. 13, pp. 2487-2504, Oct. 2005.

[10] J. N. Renner, L. F. Greenlee, K. E. Ayres, and A. M. Herring, "Electrochemical Synthesis of Ammonia: A Low Pressure, Low Temperature Approach," Interface Mag., vol. 24, no. 2, pp. 51-57, Jan. 2015.

[11] S. T. Wismann et al., "Electrified methane reforming: A compact approach to greener industrial hydrogen production," Science (80-. )., vol. 364, no. 6442, pp. 756759, May 2019.

[12] A. J. Martín, T. Shinagawa, and J. Pérez-Ramírez, "Electrocatalytic Reduction of Nitrogen: From Haber-Bosch to Ammonia Artificial Leaf," Chem, no. 2018, pp. 1-21, Nov. 2018.

[13] S. Z. Andersen et al., "A rigorous electrochemical ammonia synthesis protocol with quantitative isotope measurements," Nature, vol. 570, no. 7762, pp. 504-508, 2019.

[14] E. Skúlason et al., "A theoretical evaluation of possible transition metal electro-catalysts for $\mathrm{N} 2$ reduction," Phys. Chem. Chem. Phys., vol. 14, no. 3, pp. 1235-1245, 2012.

[15] S. Back and Y. Jung, "On the mechanism of electrochemical ammonia synthesis on the Ru catalyst," Phys. Chem. Chem. Phys., vol. 18, no. 13, pp. 9161-9166, 2016.

[16] A. R. Singh et al., "Electrochemical Ammonia SynthesisThe Selectivity Challenge," ACS Catal., vol. 7, no. 1, pp. 706-709, Jan. 2017.

[17] F. Zhou et al., "Electro-synthesis of ammonia from nitrogen at ambient temperature and pressure in ionic liquids," Energy Environ. Sci., vol. 10, no. 12, pp. 2516-2520, 2017. [18] F. Fichter, P. Girard, and H. Erlenmeyer, "Elektrolytische
Bindung von komprimiertem Stickstoff bei gewëhnlichęh

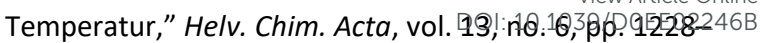
1236, Dec. 1930.

[19] A. Tsuneto, A. Kudo, and T. Sakata, "Efficient Electrochemical Reduction of N2 to NH3 Catalyzed by Lithium," Chem. Lett., vol. 22, no. 5, pp. 851-854, May 1993.

[20] A. Tsuneto, A. Kudo, and T. Sakata, "Lithium-mediated electrochemical reduction of high pressure $\mathrm{N} 2$ to NH3," J. Electroanal. Chem., vol. 367, no. 1-2, pp. 183-188, Mar. 1994.

[21] N. Lazouski, Z. J. Schiffer, K. Williams, and K. Manthiram, "Understanding Continuous Lithium-Mediated Electrochemical Nitrogen Reduction," Joule, vol. 3, no. 4, pp. 1127-1139, Apr. 2019.

[22] N. Lazouski, M. Chung, K. Williams, M. L. Gala, and K. Manthiram, "Nonaqueous gas diffusion electrodes for rapid ammonia synthesis from nitrogen and water," Nat. Catal., vol. (accepted), 2020.

[23] J. A. Schwalbe et al., "A Combined Theory-Experiment Analysis of the Surface Species in Lithium-Mediated NH3 Electrosynthesis," ChemElectroChem, vol. 7, pp. 15421549, 2020.

[24] G. K. H. Wiberg, M. J. Fleige, and M. Arenz, "Design and test of a flexible electrochemical setup for measurements in aqueous electrolyte solutions at elevated temperature and pressure," Rev. Sci. Instrum., vol. 85, no. 8, p. 085105, Aug. 2014.

[25] K. Xu, "Electrolytes and interphases in Li-ion batteries and beyond," Chem. Rev., vol. 114, no. 23, pp. 11503-11618, 2014.

[26] A. R. Singh, B. A. Rohr, M. J. Statt, J. A. Schwalbe, M. Cargnello, and J. K. Nørskov, "Strategies toward Selective Electrochemical Ammonia Synthesis," ACS Catal., vol. 9, no. 9, pp. 8316-8324, Sep. 2019.

[27] J. M. McEnaney et al., "Ammonia synthesis from N2 and $\mathrm{H} 2 \mathrm{O}$ using a lithium cycling electrification strategy at atmospheric pressure," Energy Environ. Sci., vol. 10, no. 7, pp. 1621-1630, 2017.

[28] D. Aurbach, E. Zinigrad, Y. Cohen, and H. Teller, "A short review of failure mechanisms of lithium metal and lithiated graphite anodes in liquid electrolyte solutions," Solic State lonics, vol. 148, no. 3-4, pp. 405-416, 2002.

[29] C. Graves, S. D. Ebbesen, S. H. Jensen, S. B. Simonsen, and M. B. Mogensen, "Eliminating degradation in solid oxide electrochemical cells by reversible operation," Nat. Mater., vol. 14, no. 2, pp. 239-244, 2015.

[30] M. S. Chandrasekar and M. Pushpavanam, "Pulse and pulse reverse plating-Conceptual, advantages and applications," Electrochim. Acta, vol. 53, no. 8, pp. 3313-3322, 2008.

[31] K. Domanski et al., "Migration of cations induces reversible performance losses over day/night cycling in perovskite solar cells," Energy Environ. Sci., vol. 10, no. 2, pp. 604613, 2017.

[32] C. W. Lee, N. H. Cho, K. T. Nam, Y. J. Hwang, and B. K. Min, "Cyclic two-step electrolysis for stable electrochemical conversion of carbon dioxide to formate," Nat. Commun., 
vol. 10, no. 1, pp. 1-8, 2019.

[33] J. Gopeesingh, M. A. Ardagh, M. Shetty, S. Burke, P.

Dauenhauer, and O. Abdelrahman, "Resonance-Promoted Formic Acid Oxidation via Dynamic Electrocatalytic Modulation," ACS Catal., 2020.

[34] A. C. Nielander et al., "A Versatile Method for Ammonia Detection in a Range of Relevant Electrolytes via Direct Nuclear Magnetic Resonance Techniques," ACS Catal., vol. 9, no. 7, pp. 5797-5802, Jul. 2019.

[35] J. H. Montoya, C. Tsai, A. Vojvodic, and J. K. Nørskov, "The Challenge of Electrochemical Ammonia Synthesis: A New Perspective on the Role of Nitrogen Scaling Relations," ChemSusChem, vol. 8, no. 13, pp. 2180-2186, Jul. 2015.

[36] M. C. Durrant, "Controlled protonation of ironmolybdenum cofactor by nitrogenase: a structural and theoretical analysis," Biochem. J., vol. 355, no. 3, pp. 569576, May 2001.

[37] R. Xu et al., "Artificial Interphases for Highly Stable Lithium Metal Anode," Matter, vol. 1, no. 2, pp. 317-344, Aug. 2019.

[38] R. Dabundo et al., "The Contamination of Commercial 15N2 Gas Stocks with 15N-Labeled Nitrate and Ammonium and Consequences for Nitrogen Fixation Measurements," PLoS One, vol. 9, no. 10, p. e110335, 2014.

[39] P. L. Searle, "The berthelot or indophenol reaction and its use in the analytical chemistry of nitrogen. A review," Analyst, vol. 109, no. 5, p. 549, 1984.

[40] D. D. Wagman et al., "The NBS tables of chemical thermodynamic properties. Selected values for inorganic and $\mathrm{C} 1$ and $\mathrm{C} 2$ organic substances in SI units [J. Phys. Chem. Ref. Data 11, Suppl. 2 (1982)]," J. Phys. Chem. Ref. Data, vol. 18, no. 4, pp. 1807-1812, Oct. 1989. 